\title{
DUAL TRIGONOMETRICAL SERIES
}

\author{
by C. J. TRANTER
}

(Received 19th September, 1958)

1. Introduction. In a recent joint paper with J. C. Cooke [1], we have given a method of determining the coefficients $a_{n}$ in the "dual "Fourier-Bessel series

$$
\left.\begin{array}{c}
\sum_{n=1}^{\infty} \alpha_{n}^{p} a_{n} J_{\nu}\left(\alpha_{n} r\right)=F(r) \quad(0<r<1), \\
\sum_{n=1}^{\infty} a_{n} J_{\nu}\left(\alpha_{n} r\right)=0 \quad(1<r<a),
\end{array}\right\}
$$

where $-1 \leqslant p \leqslant 1, F(r)$ is specified and $\alpha_{n}$ is a positive root of $J_{v}\left(\alpha_{n} a\right)=0$. This method reduced the problem to the solution of an infinite set of algebraical equations and it was shown that, under certain circumstances, numerical values for the coefficients could be obtained fairly readily.

By modifying the analysis (originally due to Beltrami) which I used in discussing certain dual integral equations [2], I have now found it possible to obtain closed expressions for the coefficients $a_{n}$ in equations ( 1 ) in the special cases $\nu= \pm \frac{1}{2}, p= \pm 1$. In these cases the Bessel functions are replaced by sines or cosines and it seems natural to describe the problem of this note as one in "dual " trigonometrical series although W. M. Shepherd [3], in a discussion of a similar but more restricted problem, describes it as one in trigonometrical series with mixed conditions.

The solution given is formal only and, beyond some obviously necessary conditions implied by the analysis, no attempt has been made to assign precise conditions under which the solution obtained is valid.

2. The case $\nu=\frac{1}{2}, p=1$. On writing $\nu=\frac{1}{2}, p=1, a=\pi / c, r=x / c, a_{n}=n^{\ddagger} A_{n}$ and $F(r)=(2 / \pi x)^{\dagger} c f(x)$, equations (1) become

$$
\left.\begin{array}{c}
\sum_{n=1}^{\infty} n A_{n} \sin n x=f(x) \quad(0<x<c), \\
\sum_{n=1}^{\infty} A_{n} \sin n x=0 \quad(c<x<\pi),
\end{array}\right\}
$$

and these are the basic dual trigonometrical series from which the coefficients $A_{n}$ are to be determined.

If $V(x)$ is the value of $\sum_{n=1}^{\infty} A_{n} \sin n x$ when $0<x<c$, the usual method of determining the coefficients in a sine series gives

$$
A_{n}=\frac{2}{\pi} \int_{0}^{c} V(x) \sin n x d x .
$$

Guided by the analysis previously used to solve a pair of dual integral equations [2], we assume

D 
that $\sum_{n=1}^{\infty} A_{n} \sin n x$ is continuous at $x=c$ and write

$$
V(x)=\sin \frac{1}{2} x \int_{\sin \{x \operatorname{cosec} c}^{1} \frac{\chi(s) d s}{\left(s^{2}-\sin ^{2} \frac{1}{2} x \operatorname{cosec}^{2} \frac{1}{2} c\right)^{\frac{1}{2}}} .
$$

Substituting for $V(x)$ from (4) in (3) and changing the order of integration, we obtain

$$
A_{n}=\frac{2}{\pi} \int_{0}^{1} \chi(s) d s \int_{0}^{2 \sin ^{-1}\left(8 \sin \frac{1}{2} c\right)} \frac{\sin n x \sin \frac{1}{2} x}{\left(s^{2}-\sin ^{2} \frac{1}{2} x \operatorname{cosec}^{2} \frac{1}{2} c\right)^{\frac{1}{2}}} d x \text {. }
$$

If we write $\sin \frac{1}{2} x=\alpha \sin \frac{1}{2} c$, the integral in $x$ in (5) becomes

$$
\begin{aligned}
& 2 \int_{0}^{8} \frac{\sin \left\{2 n \sin ^{-1}\left(\alpha \sin \frac{1}{2} c\right)\right\} \alpha \sin ^{2} \frac{1}{2} c}{\left\{\left(s^{2}-\alpha^{2}\right)\left(1-\alpha^{2} \sin ^{2} \frac{1}{2} c\right)\right\}^{\frac{3}{2}}} d \alpha \\
& =4 n \sin ^{3} \frac{1}{2} c \int_{0}^{8} \frac{\alpha^{2}}{\left(s^{2}-\alpha^{2}\right)^{\frac{1}{3}}} F\left(1+n, 1-n ; \frac{3}{2} ; \alpha^{2} \sin ^{2} \frac{1}{2} c\right) d \alpha,
\end{aligned}
$$

and, using the value of the definite integral in (6) as given by Magnus and Oberhettinger $[4$, p. 11], we can write equation (5) as

$$
A_{n}=2 n \sin ^{3} \frac{1}{2} c \int_{0}^{1} F\left(1+n, 1-n: 2 ; s^{2} \sin \frac{1}{2} c\right) s^{2} \chi(s) d s .
$$

The function $\chi(s)$ has now to be chosen so that $A_{n}$ satisfies the first of equations (2). Proceeding along the lines previously followed in solving dual integral equations [2], we multiply the first of equations (1) by $\sin \frac{1}{2} x$ and integrate with respect to $x$ between 0 and $x$. When the substitution $\sin \frac{1}{2} x=\rho \sin \frac{1}{2} c$ is made, this gives

$$
\sum_{n=1}^{\infty} n A_{n}\left[\frac{\sin \left\{(2 n-1) \sin ^{-1}\left(\rho \sin \frac{1}{2} c\right)\right\}}{2 n-1}-\frac{\sin \left\{(2 n+1) \sin ^{-1}\left(\rho \sin \frac{1}{2} c\right)\right\}}{2 n+1}\right]=I(\rho) \quad(0<\rho<1),
$$

where

$$
I(\rho)=\int_{0}^{2 \sin ^{-1}(\rho \sin t c)} f(x) \sin \frac{1}{2} x d x .
$$

Expressing the sines as hypergeometric functions and using the first of Gauss's recursion formulae $[4$, p. 9], we can write equation (8) in the form

$$
\sum_{n=1}^{\infty} n^{2} A_{n} F\left(1+n, 1-n ; \frac{5}{2} ; \rho^{2} \sin ^{2} \frac{1}{2} c\right)=\frac{3 I(\rho)}{4 \rho^{3} \sin ^{3} \frac{1}{2} c} \quad(0<\rho<1) .
$$

Substituting for $A_{n}$ from (7) and interchanging the order of integration and summation, we obtain

$$
\int_{0}^{1} s^{2} \chi(s) \Sigma_{1}(s, \rho, c) d s=\frac{3 I(\rho)}{8 \rho^{3} \sin ^{6} \frac{1}{2} c} \quad(0<\rho<1)
$$

where

$$
\Sigma_{1}(s, \rho, c)=\sum_{n=1}^{\infty} n^{3} F\left(1+n, 1-n ; 2 ; s^{2} \sin ^{2} \frac{1}{2} c\right) F\left(1+n, 1-n ; \frac{5}{2} ; \rho^{2} \sin ^{2} \frac{1}{2} c\right) .
$$

The value of $\Sigma_{1}(s, \rho, c)$ can be found as follows. Since [5, p. 140] 


$$
\sum_{n=1}^{\infty} n^{2} F\left(1+n, 1-n ; 2 ; s^{2} \sin ^{2} \frac{1}{2} c\right) J_{2 n}(z)=\frac{z J_{1}\left(z s \sin \frac{1}{2} c\right)}{4 s \sin \frac{1}{2} c}
$$

and $[5$, p. 401]

$$
\int_{0}^{\infty} z^{-1} J_{2 n}(z) J_{\frac{3}{3}}\left(z \rho \sin \frac{1}{2} c\right) d z=\frac{4}{3}\left(\frac{\rho^{3} \sin ^{3} \frac{1}{2} c}{2 \pi}\right)^{\frac{1}{2}} n F\left(1+n, 1-n ; \frac{5}{2} ; \rho^{2} \sin ^{2} \frac{1}{2} c\right),
$$

multiplication of (13) by $z^{-1} J_{\frac{3}{3}}\left(z \rho \sin \frac{1}{2} c\right)$, integration with respect to $z$ between 0 and $\infty$ and use of (12), (14) gives

$$
\Sigma_{1}(s, \rho, c)=\frac{3}{16 s}\left(\frac{2 \pi}{\rho^{3} \sin ^{5} \frac{1}{2} c}\right)^{\frac{1}{t}} \int_{0}^{\infty} z^{\frac{1}{1}} J_{1}\left(z s \sin \frac{1}{2} c\right) J_{\frac{3}{3}}\left(z \rho \sin \frac{1}{2} c\right) d z .
$$

Evaluating the infinite integral on the right of $(15)[5$, p. 401], we obtain

$$
\Sigma_{1}(s, \rho, c)=\left\{\begin{array}{l}
0 \quad(\rho<s), \\
\frac{3}{8} \rho^{-3}\left(\rho^{2}-s^{2}\right)^{-\frac{1}{2} \operatorname{cosec}^{4} \frac{1}{2} c} \quad(\rho>s) .
\end{array}\right\}
$$

Substitution from equation (16) in equation (11) gives

$$
\int_{0}^{\rho} \frac{s^{2} \chi(s)}{\left(\rho^{2}-s^{2}\right)^{\frac{1}{3}}} d s=\operatorname{cosec}^{2} \frac{1}{2} c I(\rho) .
$$

This is Schlömilch's integral equation and its solution [6, p. 229] is given by

$$
\frac{\pi}{2} s^{2} \chi(s)=s \operatorname{cosec}^{2}{ }_{2}^{1} c \int_{0}^{a} \frac{I^{\prime}(\rho)}{\left(s^{2}-\rho^{2}\right)^{\frac{1}{2}}} d \rho .
$$

Use of equation (9) enables this to be written

$$
\chi(s)=\frac{4}{\pi s} \int_{0}^{s} \frac{\rho f\left\{2 \sin ^{-1}\left(\rho \sin \frac{1}{2} c\right)\right\}}{\left\{\left(s^{2}-\rho^{2}\right)\left(1-\rho^{2} \sin ^{2} \frac{1}{2} c\right)\right\}^{t}} d \rho,
$$

and equations (17) and (7) provide a complete solution of the problem.

3. Example 1. As an example take $\nu=\frac{1}{2}, p=1, f(x)=\sin x$. Equation (17) gives

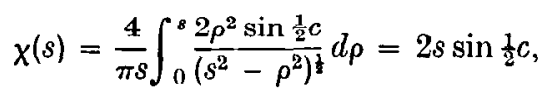

and equation (7) leads to

$$
\begin{aligned}
A_{n} & =4 n \sin ^{4} \frac{1}{2} c \int_{0}^{1} F\left(1+n, 1-n ; 2 ; s^{2} \sin ^{2} \frac{1}{2} c\right) s^{3} d s \\
& =n \sin ^{4} \frac{1}{2} c F\left(1+n ; 1-n ; 3 ; \sin ^{2} \frac{1}{2} c\right)
\end{aligned}
$$

when the definite integral is evaluated $[4$, p. 11].

At the end of his paper, Shepherd [3] gives this example for the special case in which $c=\frac{1}{2} \pi$. For this value of $c$,

$$
\begin{aligned}
2 A_{n} & =\frac{1}{2} n F\left(1+n, 1-n ; 3 ; \frac{1}{2}\right) \\
& =F\left(n, 1-n ; 2 ; \frac{1}{2}\right)-F\left(1+n,-n ; 2 ; \frac{1}{2}\right),
\end{aligned}
$$

and each of the hypergeometric functions can be expressed in terms of gamma functions through the formula [7, p. 104] 


$$
F\left(a, 1-a ; b ; \frac{1}{2}\right)=2^{1-b} \frac{\Gamma(b) \Gamma\left(\frac{1}{2}\right)}{\Gamma\left(\frac{1}{2} a+\frac{1}{2} b\right) \Gamma\left(\frac{1}{2} b-\frac{1}{2} a+\frac{1}{2}\right)} .
$$

It is then easy to verify that the value of $A_{n}$ is in agreement with that given by Shepherd.

4. The case $\nu=-\frac{1}{2}, p=1$. Here the coefficients $A_{n}$ have to be determined from the dual cosine series

$$
\left.\begin{array}{l}
\sum_{n=1}^{\infty}(2 n-1) A_{n} \cos \frac{1}{2}(2 n-1) x=f(x) \quad(0<x<c) \\
\sum_{n=1}^{\infty} A_{n} \cos \frac{1}{2}(2 n-1) x=0 \quad(c<x<\pi)
\end{array}\right\}
$$

and the solution is very similar to that given in $\S 2 . \quad V(x)$ is now the value of

$$
\sum_{n=1}^{\infty} A_{n} \cos \frac{1}{2}(2 n-1) x \quad \text { when } 0<x<c
$$

so that

$$
A_{n}=\frac{2}{\pi} \int_{0}^{c} V(x) \cos \frac{1}{2}(2 n-1) x d x .
$$

In place of (4), we now write

$$
V(x)=\int_{\sin \frac{1}{6} x \operatorname{cosec} \frac{1}{2} c}^{1} \frac{\chi(s) d s}{\left(s^{2}-\sin ^{2} \frac{1}{2} x \operatorname{cosec}^{2} \frac{1}{2} c\right)^{\frac{1}{t}}}
$$

and, working as before, we find that equations (19) and (20) lead to

$$
A_{n}=2 \sin \frac{1}{2} c \int_{0}^{1} F\left(n, 1-n ; 1 ; s^{2} \sin ^{2} \frac{1}{2} c\right) \chi(s) d s .
$$

Integrating the first of equations (18) with respect to $x$ between 0 and $x$, we obtain the equation corresponding to equation (10), namely

$$
\sum_{n=1}^{\infty}(2 n-1) A_{n} F\left(n, 1-n ; \frac{3}{2} ; \rho^{2} \sin ^{2} \frac{1}{2} c\right)=\frac{I(\rho)}{2 \rho \sin \frac{1}{2} c} \quad(0<\rho<1),
$$

where now

$$
I(\rho)=\int_{0}^{2 \sin ^{-1}(\rho \sin \varphi c)} f(x) d x .
$$

Substitution for $A_{n}$ from (21) in (22) gives

$$
\int_{0}^{1} \chi(s) \Sigma_{2}(s, \rho, c) d s=\frac{I(\rho)}{4 \rho \sin ^{2} \frac{1}{2} c} \quad(0<\rho<1),
$$

with

$$
\Sigma_{2}(s, \rho, c)=\sum_{n=1}^{\infty}(2 n-1) F\left(n, 1-n ; 1 ; s^{2} \sin ^{2} \frac{1}{2} c\right) F\left(n, 1-n ; \frac{3}{2} ; \rho^{2} \sin ^{2} \frac{1}{2} c\right) .
$$

The value of $\Sigma_{2}(s, \rho, c)$ can be found in a similar way to that of $\Sigma_{1}(s, \rho, c)$ and is 


$$
\Sigma_{2}(s, \rho, c)=\left\{\begin{array}{ll}
0 & (\rho<s), \\
\frac{1}{2} \rho^{-1}\left(\rho^{2}-s^{2}\right)^{-1} \operatorname{cosec}^{2} \frac{1}{2} c & (\rho>s) .
\end{array}\right\}
$$

From equations (24) and (26) it follows that

$$
\int_{0}^{\rho} \frac{\chi(s)}{\left(\rho^{2}-s^{2}\right)^{\frac{1}{3}}} d s=\frac{1}{2} I(\rho)
$$

with solution

$$
\frac{\pi}{2} \chi(s)=\frac{1}{2} s \int_{0}^{s} \frac{I^{\prime}(\rho)}{\left(s^{2}-\rho^{2}\right)^{t}} d \rho .
$$

Using the value of $I(\rho)$ given in (23), we obtain

$$
\chi(s)=\frac{2}{\pi} s \sin \frac{1}{2} c \int_{0}^{s} \frac{f\left\{2 \sin ^{-1}\left(\rho \sin \frac{1}{2} c\right)\right\}}{\left\{\left(s^{2}-\rho^{2}\right)\left(1-\rho^{2} \sin ^{2} \frac{1}{2} c\right)\right\}^{1}} d \rho,
$$

and equations (27) and (21) give the complete solution in this case.

5. The case $\nu=\frac{1}{2}, p=-1$. Writing $\nu=\frac{1}{2}, p=-1, a=\pi / c, r=x / c, a_{n}=n^{\dagger} A_{n}$ and $c F(r)=(2 / \pi x)^{\mathbf{t}} f(x)$, equations (1) become

$$
\left.\begin{array}{cc}
\sum_{n=1}^{\infty} \frac{A_{n}}{n} \sin n x=f(x) & (0<x<c), \\
\sum_{n=1}^{\infty} A_{n} \sin n x=0 \quad & (c<x<\pi),
\end{array}\right\}
$$

and these are the basic dual trigonometrical series from which the coefficients $A_{n}$ are to be determined.

If $\sigma(x)$ is the value of $\sum_{n=1}^{\infty} A_{n} \sin n x$ when $0<x<c$, it follows that

$$
A_{n}=\frac{2}{\pi} \int_{0}^{c} \sigma(x) \sin n x d x
$$

In line with the analysis used in discussing the analogous dual integral equations [2], we express $\sigma(x)$ in the form*

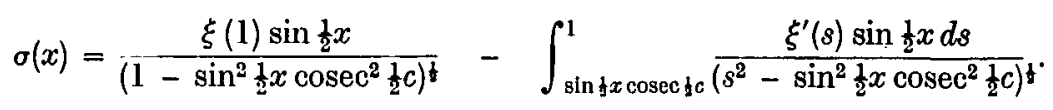

Substituting for $\sigma(x)$ from (30) in (29) and changing the order of integration, we obtain

$$
\frac{\pi}{2} A_{n}=\xi(1) \int_{0}^{c} \frac{\sin n x \sin \frac{1}{2} x d x}{\left(1-\sin ^{2} \frac{1}{2} x \operatorname{cosec}^{2} \frac{1}{2} c\right)^{\frac{1}{t}}}-\int_{0}^{1} \xi^{\prime}(s) d s \int_{0}^{2 \sin ^{-1}\left(8 \sin \frac{1}{2} c\right)} \frac{\sin n x \sin \frac{1}{2} x d x}{\left(s^{2}-\sin ^{2} \frac{1}{2} x \operatorname{cosec}^{2} \frac{1}{2} c\right)^{\frac{1}{t}}}
$$

If we write $\sin \frac{1}{2} x=\alpha \sin \frac{1}{2} c$ and work as in equations (6) and (7), the integral in $x$ on the extreme right of (5) can be written

$$
\pi n s^{2} \sin ^{3} \frac{1}{2} c F\left(1+n, 1-n ; 2 ; s^{2} \sin ^{2} \frac{1}{2} c\right) .
$$

* This form is suggested by the relations obtained by combining equations (21) and (24) of reference [2]. 
The first integral in $x$ on the right of (31) can be found by setting $s=1$ in (32), so that equation (31) gives

$\frac{A_{n}}{2 n} \operatorname{cosec}^{3} \frac{1}{2} c=\xi(1) F\left(1+n, 1-n ; 2 ; \sin ^{2} \frac{1}{2} c\right)-\int_{0}^{1} s^{2} \xi^{\prime}(s) F\left(1+n, 1-n ; 2 ; s^{2} \sin ^{2} \frac{1}{2} c\right) d s$.

Integrating by parts and using the relation $[4$, p. 10]

$$
\frac{d}{d s}\left\{s^{2} F\left(1+n, 1-n ; 2 ; s^{2} \sin ^{2} \frac{1}{2} c\right)\right\}=2 s F\left(1+n, 1-n ; 1 ; s^{2} \sin ^{2} \frac{1}{2} c\right)
$$

we can write equation (33) as

$$
\begin{aligned}
\frac{A_{n}}{4 n} \operatorname{cosec}^{3} \frac{1}{2} c & =\int_{0}^{1} s \xi(s) F\left(1+n, 1-n ; 1 ; s^{2} \sin ^{2} \frac{1}{2} c\right) d s \\
& =\int_{0}^{1} \frac{s \xi(s)}{1-s^{2} \sin ^{2} \frac{1}{2} c} F\left(n,-n ; 1 ; s^{2} \sin ^{2} \frac{1}{2} c\right) d s,
\end{aligned}
$$

the last step resulting from the transformation formula for the hypergeometric function $[4$, p. 8].

The function $\xi(s)$ has now to be chosen so that $A_{n}$ satisfies the first of equations (28). Writing $\sin \frac{1}{2} x=\rho \sin \frac{1}{2} c$ and expressing the sine as a hypergeometric function, we can write this equation as

$$
\sum_{n=1}^{\infty} A_{n} F\left(1+n, 1-n ; \frac{3}{2} ; \rho^{2} \sin ^{2} \frac{1}{2} c\right)=\phi(\rho) \quad(0<\rho<1),
$$

where

$$
\phi(\rho)=\frac{f\left\{2 \sin ^{-1}\left(\rho \sin \frac{1}{2} c\right)\right\}}{2 \rho \sin \frac{1}{2} c\left(1-\rho^{2} \sin ^{2} \frac{1}{2} c\right)} .
$$

Substituting for $A_{n}$ from (34) and interchanging the order of integration and summation, we obtain

$$
\int_{0}^{1} \frac{s \xi(s)}{1-s^{2} \sin ^{2} \frac{1}{2} c} \Sigma_{3}(s, \rho, c) d s=\frac{1}{4} \phi(\rho) \operatorname{cosec}^{3} \frac{1}{2} c \quad(0<\rho<1)
$$

where

$$
\Sigma_{3}(s, \rho, c)=\sum_{n=1}^{\infty} n F\left(n, 1-n ; 1 ; s^{2} \sin ^{2} \frac{1}{2} c\right) F\left(1+n, 1-n ; 3 ; \rho^{2} \sin ^{2} \frac{1}{2} c\right)
$$

The value of $\Sigma_{3}(s, \rho, c)$ can be found in a similar way to that used previously [equations $(13)-(16)]$ : it is

$$
\Sigma_{3}(s, \rho, c)=\left\{\begin{array}{ll}
0 & (\rho<s), \\
\frac{1}{4} \rho^{-1}\left(\rho^{2}-s^{2}\right)^{-\frac{1}{d}} \operatorname{cosec}^{2} \frac{1}{y} c & (\rho>s)
\end{array}\right\}
$$

Substitution for $\Sigma_{3}(s, \rho, c)$ from (39) in (37) now gives

$$
\int_{0}^{\rho} \frac{s \xi(s)}{\left(1-s^{2} \sin ^{2} \frac{1}{2} c\right)\left(\rho^{2}-s^{2}\right)^{\frac{1}{3}}} d s=\rho \phi(\rho) \operatorname{cosec} \frac{1}{2} c .
$$

This is Schlömilch's integral equation and, using the form of solution given by Copson [8], we 
have

$$
\frac{s \xi(s)}{1-s^{2} \sin ^{2} \frac{1}{2} c}=\frac{2}{\pi} \operatorname{cosec} \frac{1}{2} c \quad \frac{d}{d s} \int_{0}^{s} \frac{\rho^{2} \phi(\rho)}{\left(s^{2}-\rho^{2}\right)^{t}} d \rho
$$

By equation (36), this can be written

$$
\xi(s)=\frac{1-s^{2} \sin ^{2} \frac{1}{2} c}{\pi s \sin ^{2} \frac{1}{2} c} \frac{d}{d s} \int_{0}^{s} \frac{\rho f\left\{2 \sin ^{-1}\left(\rho \sin \frac{1}{2} c\right)\right\}}{\left\{\left(s^{2}-\rho^{2}\right)\left(1-\rho^{2} \sin ^{2} \frac{1}{2} c\right)\right\}} d \rho,
$$

and the complete solution is given by equations (40) and (34).

6. The case $\nu=-\frac{1}{2}, p=-1$. Here the coefficients $A_{n}$ have to be determined from the dual cosine series

$$
\left.\begin{array}{l}
\sum_{n=1}^{\infty} \frac{A_{n}}{2 n-1} \cos \frac{1}{2}(2 n-1) x=f(x) \quad(0<x<c), \\
\sum_{n=1}^{\infty} A_{n} \cos \frac{1}{2}(2 n-1) x=0 \quad(c<x<\pi),
\end{array}\right\}
$$

and the solution again proceeds in a similar way. $\sigma(x)$ is now the value of

$$
\sum_{n=1}^{\infty} A_{n} \cos \frac{1}{2}(2 n-1) x \quad \text { when } 0<x<c
$$

so that

$$
A_{n}=\frac{2}{\pi} \int_{0}^{c} \sigma(x) \cos \frac{1}{2}(2 n-1) x d x
$$

In place of $(30)$, we now write

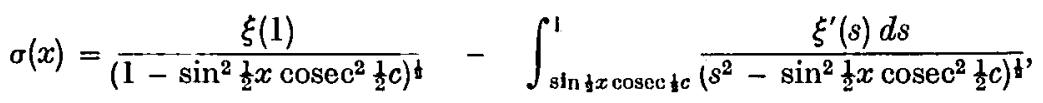

and, working as before, we find that equations (42) and (43) lead to

$$
\frac{1}{2} A_{n} \operatorname{cosec} \frac{1}{2} c=\xi(1) F\left(n, 1-n ; 1 ; \sin ^{2} \frac{1}{2} c\right)-\int_{0}^{1} \xi^{\prime}(s) F\left(n, 1-n ; 1 ; s^{2} \sin ^{2} \frac{1}{2} c\right) d s .
$$

To enable the solution in this case to be reduced to that of Schlömilch's integral equation, a slightly different procedure is now adopted. The first of equations (41) is differentiated with respect to $x$ before making the substitution $\sin \frac{1}{2} x=\rho \sin \frac{1}{2} c$. This leads to

$\rho \sin \frac{1}{2} c \sum_{n=1}^{\infty}(2 n-1) A_{n} F\left(n, 1-n ; \frac{3}{2} ; \rho^{2} \sin ^{2} \frac{1}{2} c\right)=-2 f^{\prime}\left\{2 \sin ^{-1}\left(\rho \sin \frac{1}{2} c\right)\right\} \quad(0<\rho<1)$.

Substitution for $A_{n}$ from (44) in (45) gives

$$
\xi(1) \Sigma_{2}(1, \rho, c)-\int_{0}^{1} \xi^{\prime}(s) \Sigma_{\mathbf{2}}(s, \rho, c) d s=-\frac{f^{\prime}\left\{2 \sin ^{-1}\left(\rho \sin \frac{1}{2} c\right)\right\}}{\rho \sin ^{2} \frac{1}{2} c} \quad(0<\rho<1),
$$

where

$$
\Sigma_{2}(s, \rho, c)=\sum_{n=1}^{\infty}(2 n-1) F\left(n, 1-n ; 1 ; s^{2} \sin ^{2} \frac{1}{2} c\right) F\left(n, 1-n ; \frac{3}{2} ; \rho^{2} \sin ^{2} \frac{1}{2} c\right) .
$$


On using the value of $\Sigma_{2}(s, \rho, c)$ given by equation (26), equation (46) can be written

$$
\int_{0}^{\rho} \frac{\xi^{\prime}(s)}{\left(\rho^{2}-s^{2}\right)^{\frac{1}{3}}} d s=2 f^{\prime}\left\{2 \sin ^{-1}\left(\rho \sin \frac{1}{2} c\right)\right\}
$$

Again using the form of solution of Schlömilch's integral equation given by Copson [8], we obtain

$$
\xi^{\prime}(s)=\frac{4}{\pi} \cdot \frac{d}{d s} \int_{0}^{s} \frac{\rho f^{\prime}\left\{2 \sin ^{-1}\left(\rho \sin \frac{1}{2} c\right)\right\}}{\left(s^{2}-\rho^{2}\right)^{\frac{1}{2}}} d \rho,
$$

and hence

$$
\xi(s)=\frac{4}{\pi} \int_{0}^{8} \frac{\rho f^{\prime}\left\{2 \sin ^{-1}\left(\rho \sin \frac{1}{2} c\right)\right\}}{\left(s^{2}-\rho^{2}\right)^{\frac{1}{2}}} d \rho+C,
$$

where $C$ is a constant. This constant can be determined by substituting from (48) in (44) and then choosing $C$ so that the first of equations (41) is satisfied. Once $C$ has been found in this manner, equations (48) and (44) give the solution to this case.

7. Example 2. As a second example take $\nu=-\frac{1}{2}, p=-1, f(x)=$ constant $=f(0)$. In this case, $f^{\prime}(x)=0$ and equation (48) gives $\xi(s)=C$. Equation (44) gives

$$
\begin{aligned}
& A_{n}=2 C \sin \frac{1}{2} c F\left(n, 1-n ; 1 ; \sin ^{2} \frac{1}{2} c\right) \\
& =2 C \sin \frac{1}{2} c P_{n-1}(\cos c) \text {, }
\end{aligned}
$$

when use is made of the expression for the Legendre polynomial as a hypergeometric function. The first of equations (41) then yields, with $x=0$,

$$
2 C \sin \frac{1}{2} c \sum_{n=1}^{\infty} \frac{P_{n-1}(\cos c)}{2 n-1}=f(0) .
$$

The sum of the series in equation (50) can be found as follows. Since

$$
\sum_{n=1}^{\infty} P_{n-1}(\cos c) h^{2 n-2}=\left(1-2 h^{2} \cos c+h^{4}\right)^{-1}
$$

it follows that

$$
\sum_{n=1}^{\infty} \frac{P_{n-1}(\cos c)}{2 n-1}=\int_{0}^{1} \frac{d h}{\left(1-2 h^{2} \cos c+h^{4}\right)^{1}}
$$

By writing $y=2 h /\left(1+h^{2}\right)$, the integral on the right of equation (51) is easily transformed into the integral

$$
\frac{1}{2} \int_{0}^{1} \frac{d y}{\left\{\left(1-y^{2}\right)\left(1-y^{2} \cos ^{2} \frac{1}{2} c\right)\right\}}
$$

and this is $\frac{1}{2} K\left(\cos \frac{1}{2} c\right)$ in the usual notation of elliptic integrals. Equations (50), (51) now provide the value of the constant $C$ and equation (49) gives

$$
A_{n}=2 \frac{P_{n-1}(\cos c)}{K\left(\cos \frac{1}{2} c\right)} f(0)
$$

I am indebted to Professor J. C. Cooke for some very helpful conversations. 


\section{REFERENCES} press).

1. J. C. Cooke and C. J. Tranter, Dual Fourier-Bessel series, Quart. J. Mech. Appl. Math. (in 60-66.

2. C. J. Tranter, On some dual integral equations, Quart. J. of Math. (Oxford) (2), 2 (1951),

3. W. M. Shepherd, On trigonometrical series with mixed conditions, Proc. London Math. Soc. (2), 43 (1937), 366-375.

4. W. Magnus and F. Oberhettinger (translated by J. Wermer), Special functions of mathematical physics (New York, 1949).

5. G. N. Watson, Theory of Bessel functions (Cambridge, 1944).

6. E. T. Whittaker and C. N. Watson, Modern analysis (Cambridge, 1920), 229.

7. A. Erdélyi, Higher transcendental functions (New York, 1953), Vol. 1.

8. E. 'T. Copson, On the problem of the electrified dise, Proc. Edinburgh Math. Soc. (3), 8 (1947), $14-19$.

Royal Military College of Science

SHRTVENHAM 